\title{
DEMOCRACIA Y CONSTITUCIÓN
}

\author{
Carlos Ruiz Schneider ${ }^{1}$
}

\section{Resumen/Abstract}

El presente ensayo defiende brevemente -en base a los trabajos de Bruce Ackerman y Jeremy Waldronla prioridad de la democracia como fundamento de la Constitución Política, a diferencia de lo que se ha denominado "fundacionalismo de los derechos". En segundo lugar discute una visión de la democracia directa - como la de Sartre- que deja de lado las instituciones y la representación a partir de elecciones como momento democrático. El artículo concluye, en base a argumentos de Chantal Mouffe y Elías Canetti, en la necesidad de un momento representativo, electoral en la democracia, para descartar la violencia.

Palabras clave: constitución, democracia, democracia directa, elecciones

\section{DEMOCRACY AND CONSTITUTION}

This paper argues for the priority of democracy as a foundation of a Political Constitution, based on the works of Bruce Ackerman and Jeremy Waldron, opposing what has been called "rights foundationalism". It discuss, in the second place, visions of direct democracy such as Sartre's, which radically criticizes free elections as elements of democracy. In the third place, the essay concludes, on the basis of Chantal Mouffe's and Elias Canetti's arguments, on the necessity of representation and free elections, in order to exclude violence in a democracy.

Keywords: constitution, democracy, direct democracy, free elections

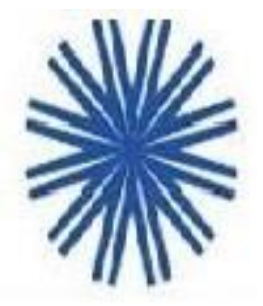

Quiero centrar mi exposición en un solo punto, la defensa y justificación de la democracia a partir de las reflexiones de varios autores.

En los debates de estos días sobre la necesidad de un cambio constitucional se ha expresado, por ejemplo, por el profesor Carlos Peña que "un acuerdo constitucional es por definición, contra mayoritario, en el sentido que supone convenir reglas que pondrán obstáculos a la

\footnotetext{
${ }^{1}$ Chileno, Universidad de Chile. E-mail: cruiz@udechile.cl
} 
futura voluntad de las mayorías. Las sociedades cuentan con constituciones para asegurarse de que ciertos aspectos de la vida social -los derechos de las personas, la configuración del estado- se erijan como momentos de incondicionalidad, aspectos que escapan a la voluntad de las mayorías. En otras palabras, la función básica de una constitución es apartar ciertas decisiones del proceso democrático.” (El Mercurio 21-XI-19).

Creo que en este punto Peña se equivoca, aunque estoy consciente de que usa este argumento para un propósito distinto de la generalidad en la que yo lo considero.

Y esto, en primer lugar porque, como lo sostiene el jurista Jeremy Waldron, "una teoría que sostenga que la mayoría abusará siempre de su poder no podría ser usada como argumento en favor de la protección de derechos. Esta teoría sería demasiado pesimista porque excluiría la posibilidad de que una mayoría pudiera iniciar y sostener restricciones constitucionales sobre ella misma, excepto por accidente.” (1991: 406). Lo que tiene que hacer un teórico que es más optimista acerca de la posibilidad de proteger derechos en una democracia es la de "[...] ser capaz de convencer a un número suficiente de sus conciudadanos, de que el respeto por los derechos es importante." (407). Como lo dice el filósofo francés Jean-Fabien Spitz comentando estas ideas de Waldron, "si la voluntad colectiva no es capaz de establecer derechos que limiten la extensión de su "propio poder, entonces esa limitación del poder es conceptualmente imposible y carece de validez" a lo que agrega que "La idea de que podría haber derechos contra la voluntad permanente y deliberada de la mayoría carece de sentido y de alcance práctico" (295). No hay axiomas autoevidentes en el derecho, aunque se crea que las ideas que provienen de Locke o Kant lo son.

Pero en segundo lugar porque como lo sostenía el jurista estadounidense Bruce Ackerman en su notable libro We the people (1991), en el caso de los Estados Unidos, cuya constitución analiza, "la Constitución pone a la democracia primero, aunque no de la manera simple que supone el monista" (16)" y que "la Constitución es primero democrática y en segundo lugar, protectora de derechos" porque "la protección judicial de los derechos depende de una afirmación democrática previa. (13).

La idea de Ackerman es que la filosofía de la Constitución americana se opone, tanto a una concepción monista de la democracia, como a una visión que identifica como "fundamentalista de los derechos ", como la de Ronald Dworkin, por ejemplo.

Construye su visión exponiendo lo que llama una concepción dualista de la democracia, que distingue dos tipos diferentes de decisiones político-jurídicas: las decisiones que toma el pueblo en lo que ha 
denominado los "momentos constitucionales" -que la Constitución de 1787 identifica con decisiones de "We, the people", pero de los que hay varios otros en la historia norteamericana, como el del New Deal, por ejemplo-, y las decisiones legales cotidianas de los Congresos, como los presupuestos o las leyes del tránsito.

Sin embargo, para Ackerman, ambos tipos de decisión son democráticos. Las primeras son mucho más exigentes, no necesariamente en términos de las mayorías requeridas, sino también respecto a la profundidad de los temas en debate, que muchas veces conciernen también a profundas opciones morales, de los tiempos que estos requieren y de la participación necesariamente muy ampliada de los ciudadanos movilizados que es necesaria. Las segundas, en cambio, son normalmente decididas por mayoría parlamentarias, y tocan temas que raras veces dejan de ser importantes, pero que podrían, incluso, hasta no serlo, como lo sostiene por ejemplo, Herbert Hart.

En el lenguaje de Dworkin, esto permite que los derechos fundamentales y constitucionales "triunfen sobre la democracia”, pero esto es sólo posible porque hay una primacía de la democracia que autoriza que estos derechos tengan un régimen especial.

No hay en los Estados Unidos, dice Ackerman derechos que el pueblo esté sólo obligado a aceptar, como las normas constitucionales "fundamentalistas" que surgen en otros países.

\section{II}

Pero esta justificación de la prioridad de la democracia nos fuerza también a hacernos preguntas sobre la democracia misma, porque no podemos negar que hoy tiene lugar un difundido cuestionamiento de este sistema político en Chile y en el mundo. En Chile esto tiene que mucho que ver con una Constitución "tramposa", como lo ha mostrado muy bien Fernando Atria, y con una carta fundamental basada en el miedo, como muestran Pablo Ruiz-Tagle y Renato Cristi. Pero también con un modelo elitista, consensual y tecnocrático de la política y la democracia, profundamente desconfiado de la "capacidad política" del pueblo.

Hay muchas críticas que se puede hacer a estos modelos, presentes en muchas esferas de la sociedad chilena, como la educación, por ejemplo. Pero más allá de ellas, me parece importante considerar una crítica de mayor envergadura a la democracia - una democracia que llama indirecta- y que desarrolla el 
filósofo francés Jean-Paul Sartre poco después de mayo de 1968 en Francia. La visión de Sartre no forma parte de las visiones "clásicas" sobre democracia en los libros sobre el tema, pero, a mi juicio, incluye una reflexión muy profunda sobre la idea de democracia directa, la rebelión popular y la revuelta, reivindicada por los grupos maoístas en Francia, con los que Sartre estuvo ligado a fines de los 1960 y que parece importante también en nuestra situación actual.

En 1973, en el momento de unas elecciones en Francia, Sartre escribe un ensayo que me parece notable, que está publicado en Situación X y que titula "Elecciones: trampa para tontos", que tiene como tema un dispositivo que consideramos central en la democracia, las elecciones y los procesos de votación.

El texto retoma los argumentos de la Crítica de la Razón Dialéctica, de 1960, y me parece que puede servir de una excelente introducción a aspectos importantes de esta obra difícil e importante. Es, además, me parece, una colección de notas y observaciones muy profundas sobre la historia política y la historia de las elecciones y el voto, sobre todo en Francia, pero no sólo en Francia, en los siglos XIX y XX.

La Crítica, para partir por ella, es un texto que desarrolla un brillante análisis dialéctico que busca mostrar cómo se construyen los grupos históricos a partir de la praxis de los individuos; es un intento por construir lo colectivo a partir del individuo. Un tipo de individualismo metodológico que por eso es altamente valorado por filósofos políticos de la tradición analítica, como Jon Elster.

El propósito de Sartre es construir un camino de racionalidad dialéctica, que no separa la racionalidad individual y la de los grupos y las orientaciones normativas, que no separa los hechos de los valores.

Eso lo lleva, en un procedimiento complejo, a mostrar que los grupos históricos se construyen a partir de la negación de la serialidad, forma que Sartre identifica con lo que llama lo práctico-inerte, caracterizado por relaciones externas. La dificultad es que estos grupos no pueden darle permanencia a esta salida de la externalidad y de lo serial hacia la comunidad, que Sartre llama fraternidad, sin el Terror. De hecho Sartre se refiere a este movimiento dialéctico como Fraternidad-Terror.

Es evidente, como lo muestran estas breves notas, que la Revolución Francesa es el ejemplo histórico más importante en los análisis sartrianos. El paso siguiente en este desarrollo dialéctico es el avance desde la Fraternidad-Terror hacia la construcción de organizaciones y partidos populares que conformarán la base de la burocracia que caracteriza a los Estados socialistas. Esta nueva argumentación lleva a Sartre a mostrar la "racionalidad" de muchos fenómenos de estos estados, como el "culto a la personalidad", al que no condena moralmente, sino que analiza como una deriva a partir del desarrollo dialéctico. Dice por 
ejemplo en un pasaje en una de las secciones finales de la Crítica: "El terror burocrático y el culto de la personalidad manifiestan una vez más la relación entre la dialéctica constituyente y la dialéctica constituida, es decir, la necesidad para una acción común en tanto que tal (y través de la diferenciación múltiple de las tareas) de reflejarse prácticamente sobre si misma para controlarse y unificarse sin cesar bajo la forma insuperable de la unidad individual. Es cierto que Stalin era el Partido y el Estado. O más bien que el Partido y el Estado eran Stalin. Pero las violencias de éste traducen, en un proceso definido la contradicción violenta de las dos dialécticas [...]” (630).

Este es, dicho de una manera casi telegráfica, el marco del pensamiento de Sartre sobre la serialidad, la fraternidad y el terror. Pero volvamos ahora al ensayo sobre las elecciones. Difícil resumirlo, pero podemos partir por algunas de las distinciones que lo estructuran, para llegar a lo que nos interesa.

La primera es la distinción entre el sufragio restringido y censitario que rige desde la Revolución Francesa en 1789 y el sufragio universal, proclamado por la Convención en 1793, que universaliza los derechos propietarios a todos, sin que esto signifique democracia directa popular: la paradoja es que los trabajadores votan en calidad de propietarios aunque carecen de propiedad. En 1848 se constituye, en la calle, según Sartre, un poder obrero fortalecido por instituciones que resguardan derechos sociales, como los Talleres Nacionales y que se opone a la Cámara elegida por sufragio universal.

El desenlace -dice Sartre- es conocido: “en mayo-junio (y luego con el golpe de Estado de Luis Napoleón Bonaparte), la legalidad masacra a la legitimidad. Frente a la legítima Comuna de Paris, la muy legal Asamblea de Bordeaux, trasladada a Versalles, imitó aquel ejemplo". Esta es la segunda oposición conceptual del texto, legalidad versus legitimidad, la que se articula con democracia indirecta versus democracia directa.

Esto es lo que vamos a hacer, recuerda Sartre, mañana, en marzo de 1973, cuando votemos: "vamos a sustituir una vez más el poder legítimo por el poder legal. Este, de una claridad aparentemente perfecta, atomiza a los votantes en nombre del sufragio universal. Aquel es todavía embrionario, difuso, oscuro en sí mismo: por ahora se confunde con el vasto movimiento antijerárquico y libertario que se halla en todas partes pero aún no está organizado. Todos los electores participan de los grupos más diversos. Pero la urna los espera no como miembros de un grupo, sino como ciudadanos. El cuarto oscuro..."es el símbolo de todas las traiciones que el individuo puede cometer hacia los grupos en que participa". Este momento corresponde a lo que en la Crítica llama la serialidad; la serialidad une a las personas, pero como Otros para sí mismos y para los demás (la cola de espera del bus, los telespectadores, etc.) (197). 
El sufragio universal es una institución que atomiza o serializa a los hombres concretos. Ellos "no pueden en ningún caso, mientras siguen en la situación serial constituir ese grupo soberano del que se nos dice que emanan todos los poderes, el Pueblo. Porque como lo hemos visto, se les ha otorgado el sufragio universal para atomizarlos e impedirles agruparse entre sî́ (199). Y continúa subrayando la experiencia que lo marca y desde la que escribe: "Es lo que se vio en junio de 1968, cuando de Gaulle pidió a Francia de pie y agrupada que votara, es decir que se postrara y se revolcara en la serialidad.” (200). Y concluye llamando a luchar contra la democracia indirecta que gobierna y que es una forma de impedir la construcción de los grupos soberanos, democráticos, y la fraternidad,

III

Hasta aquí un resumen del texto de Sartre. Creo que es un análisis profundo y que hay que tomar en cuenta, porque efectivamente no nos basta con la democracia representativa. O indirecta. La democracia que queremos deberá incorporar también elementos de democracia directa o participativa.

El análisis de Sartre tiene, a mi juicio, dos aportes muy interesantes. Puesto que su ejemplo central de una revolución es la Revolución Francesa, se refiere a la racionalidad de la acción de los grupos y las instituciones, sin la mediación de los partidos políticos, constituidos durante el siglo XIX (salvo, cuando se refiere posteriormente a ejemplos de los partidos comunistas y a las sociedades socialistas), cuestión que hace sus descripciones más actuales. En segundo lugar, la dialéctica de Sartre, precisamente porque parte de la praxis individual y su camino hacia la construcción de los grupos revolucionarios y la historia, implica una visión "desde abajo", por decirlo así -aunque sea desde los individuos-y no desde el poder constituido, lo que cuestiona y fragiliza la percepción de las estructuras tradicionales del poder y especialmente al Estado, visto como un resultado de las contradicciones entre los grupos y como freno provisorio de los avances de la rebelión popular. Esto es muy diferente de una visión estructuralista de la sociedad como la de Althusser, por ejemplo, que parte desde las relaciones de producción, el estado y sus aparatos y concluye en la sujeción de los sujetos. Por esto en Sartre, el tema de la ideología, por ejemplo, es relativamente menos importante: el objetivo de Sartre es pensar la revolución, no la sujeción de los sujetos a las estructuras. Y esto porque le parece que es desde la perspectiva de la revolución, que aparece la verdad inestable y frágil de las estructuras. 
Pero hay varios puntos de su argumento que me parecen equivocados. En primer lugar, creo que están profundamente marcados por el contexto de una democracia representativa relativamente establecida, como es el caso de Francia, por lo menos desde comienzos de siglo hasta 1940, y luego desde 1945 hasta de Gaulle, y también después, aunque con rasgos autoritarios más marcados. Y esto hace que su reducción del acto de votar a un comportamiento serial no describa bien otras experiencias y otros contextos de elecciones democráticas como por ejemplo, la que vivimos en Chile en los años 1988 y 1989. En ese momento, para nosotros las elecciones libres y el acto de votar eran acciones llenas de significado y no comportamientos práctico-inertes y seriales, como llama Sartre a las elecciones. Diríamos más bien que, frente a la dictadura chilena, eran exactamente lo contrario de lo serial: Nos constituimos, en parte en base a ellas, como grupo (en el sentido sartriano), como negación del Otro atomizado y amenazante que la dictadura nos hacía ver en cada uno de nuestros vecinos, por ejemplo. Es cierto que ese impulso fue parcialmente ahogado por las instituciones de la transición que sí construyeron una forma de elitización y serialización de la democracia y la política, pero eso no le quita nada a la experiencia colectiva de fines de los 80 , nucleada en torno a las elecciones y la reconquista de la democracia.

\section{IV}

Más allá de nuestro ejemplo chileno, en uno de sus libros recientes, En torno a lo Político, Chantal Mouffe, a partir de una reflexión de Elías Canetti, destaca bien lo que está en juego con las elecciones y con el voto.

Chantal Mouffe quiere mostrarnos, con la cita de Canetti, el papel de ciertas prácticas o hechos institucionales como el voto, que nos permiten pasar de la lógica schmittiana de amigo-enemigo y la guerra social, a la lógica agonista propia de la política.

Dice Mouffe en su texto que "En unas pocas páginas brillantes del capítulo "Masa e Historia" de Masa y Poder, dedicadas a analizar la naturaleza del sistema parlamentario, Canetti señala que tal sistema utiliza la estructura psicológica de ejércitos adversarios, y representa una forma de guerra en la que se ha renunciado a matar. Y cita algunos textos de Canetti, como el siguiente:

En una votación parlamentaria todo cuanto hay que hacer es verificar la fuerza de ambos grupos en un lugar y momento determinados. No basta con conocerla de antemano. Un partido puede tener trescientos sesenta delegados y el otro sólo doscientos cuarenta: la votación sigue siendo decisiva en tanto instante en que se miden realmente las fuerzas. Es el vestigio del choque cruento, que cristaliza de diversas maneras, incluidas 
amenazas, injurias y una excitación física que puede llegar a las manos, incluso al lanzamiento de proyectiles. Pero el recuento de votos pone fin a la batalla. (Mouffe 2007: 2) (Canetti 2018: 269).

Y agrega:

La solemnidad de todas estas operaciones proviene de la renuncia a la muerte como instrumento de decisión. Con cada una de las papeletas la muerte es, por decirlo así descartada. Pero lo que ella habría logrado, la liquidación de la fuerza del adversario, es escrupulosamente registrado en un número. Quien juega con estos números, quien los borra o falsifica, vuelve a dar lugar a la muerte sin darse cuenta" (Mouffe: 29) (Canetti: 272).

Esto es lo que sucede, según Canetti, también “con quienes se burlan de las papeletas de voto...(Estas) como los tratados, son para ellos meros jirones de papel. El que no estén empapados en sangre les parece despreciable" (Canetti: 272).

Para Chantal Mouffe, la reflexión de Canetti “es un ejemplo excelente de cómo los enemigos pueden ser transformados en adversarios, y aquí vemos claramente cómo los conflictos pueden establecerse de un modo que no es antagónico sino agonista" (29). Esto no es un tema de elección racional, ni de progreso a través de la deliberación y la razón, destaca Mouffe, y nuevamente se apoya en Canetti :

Nadie ha creído nunca - dice éste-que la opinión de la mayoría sea ...la más sensata. Una voluntad se opone a otra, como en una guerra; cada una de estas voluntades está convencida de tener la razón y la sensatez de su parte... El sentido de un partido consiste justamente en mantener despiertas esa voluntad y esa convicción. El adversario derrotado en la votación no se resigna porque deje de creer en sus derechos, simplemente se da por vencido. Le resulta fácil darse por vencido pues nada le sucede. De ninguna manera es castigado por su anterior actitud hostil. Si se tratase de poner su vida en juego, reaccionaría de manera muy distinta. Pero cuenta con futuras batallas. Su número no tiene límite fijado y no es muerto en batalla alguna. (Canetti: 270).

Según Mouffe, Canetti nos permite comprender la importancia del sistema parlamentario -y de las legislaturas, (diríamos)- en la "construcción de un nosotros/ellos compatible con el pluralismo democrático". Y continúa diciendo que "Cuando las instituciones parlamentarias son destruidas o debilitadas, la posibilidad de una confrontación agonista desaparece y es reemplazada por un nosotros/ ellos antagónico". (Mouffe: 30). Es decir, por la violencia y la guerra civil.

Me parece que este análisis de Mouffe fundado en Canetti, es útil hoy para nosotros en muchos sentidos. En primer lugar para iluminarnos sobre uno de los sentidos profundos de instituciones y prácticas como 
el voto. Y hay otros fundamentales como la igualdad de los que votan, que lo conecta profundamente con la democracia. En segundo lugar, porque nos permite ver también lo que está en juego, lo que nos amenaza, cuando descalificamos a estas instituciones "formales" y la política: el desarrollo de derivaciones y prácticas autoritarias o totalitarias.

\section{Referencias bibliográficas}

Carlos Peña (2019), “El acuerdo constitucional”, El Mercurio 21-XI.

Jeremy Waldron (1991), "Rights and majorities. Rousseau revisited." en Liberal Rights. Collected Papers (1981-1991), Cambridge, Cambridge University Press.

Jean-Fabien Spitz (2001), "La conception dworkinienne de la démocratie et ses critiques", Archives de la Philosophie du Droit.

Bruce Ackerman(1991), We the people. I Foundations, Harvard University Press, 1991. (1989) “Constitutional politics/ constitutional law" Yale Law Journal, Vol 99, N³.

Jean-Paul Sartre (1977), "Elecciones: trampa para tontos" en Situaciones X. Autorretrato a los 70 años, Buenos Aires, Losada. (1961), Critique de la raison dialectique, Paris, Gallimard.

Jean-Paul Sartre, Pierre Victor, Philippe Gavi (1974), On a raison de se révolter, Paris, Gallimard.

Alain Badiou (2009) “Jean-Paul Sartre 1905-1980” en Pequeño panteón portátil, EFE.

Sophie Wahnich (2016), » En quoi la fraternité est- elle révolutionnaire. Sartre et Saint-Just en regard", en Jordi Riba, y Patrice Vermeren, , La Fraternité Réveillée, Paris L'Harmattan.

Chantal Mouffe (2007), En torno a lo político, Buenos Aires, Fondo de Cultura Económica.

Elias Canetti (2018), Masa y poder, Madrid, Alianza Editorial. 